\title{
Observation of Peptide-Ion Generation by Laser-Induced Surface Heating from Tungsten Silicide Surfaces
}

\author{
Shin Hye Kim ${ }^{\mathrm{a}, \mathrm{b}}$, Sun Hwa Park ${ }^{\mathrm{c}, \mathrm{d}}$, Jae Yong Song ${ }^{\mathrm{c}}$, and Sang Yun Han ${ }^{\mathrm{a}, *}$ \\ ${ }^{a}$ Center for Nano-Bio Technology, Korea Research Institute of Standards and Science, Daejeon 305-340, Republic of Korea \\ ${ }^{b}$ Department of Chemistry, Chung-Nam National University, Daejeon 305-764, Republic of Korea \\ ${ }^{c}$ Center for Nanocharacterization, Korea Research Institute of Standards and Science, Daejeon 305-340, Republic of Korea \\ ${ }^{d}$ Department of Nanomaterials Science and Engineering, University of Science and Technology, Daejeon 305-350, Republic of \\ Korea
}

Received February 27, 2012; Accepted March 6, 2012

First published on the web March 15, 2011; DOI: 10.5478/MSL.2012.3.1.018

\begin{abstract}
We report observation of laser desorption/ionization (LDI) of peptides from flat surfaces of tungsten silicide (WSi ${ }_{2}$ ). In contrast to MALDI (matrix-assisted laser desorption/ionization) and SALDI (surface-assisted laser desorption/ionization) mass spectrometry, this study did not utilize any matrices and surface nanostructures. In this work, LDI on WSi ${ }_{2}$ surfaces is demonstrated to cover a mass range up to 1,600 Da (somatostatin; monoisotopic mass = 1637.9 Da). In addition, it exhibited a high sensitivity, which could detect peptides, which could detect peptides of low femtomole levels (20 fmol for angiotensin II). The observed LDI process was discussed to be largely thermal, more specifically, due to laser-induced surface heating that is most likely promoted by the low thermal diffusivity $(\kappa)$ of $\mathrm{WSi}_{2}$ substrate.
\end{abstract}

Key words: Laser desorption/ionization (LDI), Tungsten silicide ( $\left.\mathrm{WSi}_{2}\right)$, Laser-induced surface heating

\section{Introduction}

Matrix-assisted laser desorption/ionization (MALDI) is a powerful means of soft ionization for mass spectrometry of thermally labile biomolecules. However, the use of organic crystals as matrix creates certain limitations on applications. It includes interference of matrix peaks in the small mass region $(<500 \mathrm{Da})$ and sweet spots. Careful matrix selection for given analytes is also important for successful mass analysis. To address such issues, extensive efforts were made to develop matrix-free laser desorption/ionization (LDI) in recent years, which can be an alternative method of soft ionization to the MALDI method. ${ }^{1}$

DIOS (desorption/ionization on porous silicon) mass spectrometry was the first demonstration of LDI, which utilized specially prepared $\mathrm{Si}$ surfaces. ${ }^{2}$ In DIOS mass spectrometry, LDI of small molecules was found to occur on the nanoporous surfaces of crystalline $\mathrm{Si}(\mathrm{c}-\mathrm{Si})$ formed by photo-electrochemical etching. However, without such surface nanostructures of a sub-micrometer dimension, the DIOS process was hardly observed to take place. Since the advent of DIOS mass spectrometry, surfaces possessing various nanostructures have been examined for their LDI capabilities. In the researches, Si-based nanostructures were most popularly investigated, the examples of which include

*Reprint requests to Dr. Sang Yun Han

E-mail: sanghan@kriss.re.kr porous Si surfaces fabricated by etching, ${ }^{3}$ clathrate-structured Si surfaces, ${ }^{4}$ Si nanowires, ${ }^{5}$ Si microcolumn and nanoposts arrays. ${ }^{6,7}$ In addition to $\mathrm{Si}$, nanostructured surfaces made of other materials, such as nanostructured Au thin film, ${ }^{8}$ selfassembled germanium nanodots, ${ }^{9}$ and zinc oxide nanowires, ${ }^{10}$ also exhibited a promising level of sensitivity and mass range for mass spectrometry. Likewise, utilization of surface nanostructures has been a key component in the previous developments of high-efficiency LDI surfaces.

On the other hand, few cases of LDI occurring on flat surfaces are known, i.e. surfaces without nanostructures, which include amorphous $\mathrm{Si}(\mathrm{a}-\mathrm{Si})$ and commercial $\mathrm{Al}$ foils. ${ }^{11,12}$ Obviously, flat surfaces for LDI have certain advantages. As it does not require fabrication of complex nanostructures on surfaces, it is easier to prepare in a large scale and even often commercilly available. By avoiding sweet spots caused by uneven matrix crystals and surface nanostructures, flat surfaces may offer an opportunity for quantitative measurement as well. In addition, lack of complexity from surface nanostructures provides a further opportunity to look into the LDI mechanism more closely. In this letter, we report LDI of peptides from flat surfaces of tungsten silicide $\left(\mathrm{WSi}_{2}\right)$.

\section{Experimental}

\section{Preparation of $\mathrm{WSi}_{2}$ surfaces}

$\mathrm{WSi}_{2}$ on $\mathrm{Si}(001)$ substrates were prepared by the $\mathrm{Ar}^{+}$ 
magnetron sputtering method. During the sputtering process, the flow of Ar gas was maintained at 50 mTorr and the working pressure was 1.7 mTorr. Various thicknesses of $\mathrm{WSi}_{2}$ were examined, including $65 \mathrm{~nm}, 260 \mathrm{~nm}$, and 400 $\mathrm{nm}$. For additional heat treatment, the samples were heated at $873 \mathrm{~K}$ in vacuum $\left(2 \times 10^{-6}\right.$ Torr $)$ for $1 \mathrm{hr}$. The prepared $\mathrm{WSi}_{2}$ surfaces were then characterized using a fieldemission scanning electron microscope (FESEM, S-4800, Hitachi), an X-ray diffractometer $(\theta-2 \theta, \mathrm{Cu}-\mathrm{K} \alpha$, Bruker D8), and an atomic force microscopy (AFM, XE-150, PSIA). The AFM (r.m.s.) roughness of $\mathrm{WSi}_{2}$ surfaces as prepared was measured to be about $0.07 \mathrm{~nm}$, and it slightly increased to be $0.2 \mathrm{~nm}$ after heat treatment. In the XRD study, $\mathrm{WSi}_{2}$ used in this work was characterized to carry a polymorphism with tetragonal and hexagonal crystal structures.

\section{LDI mass spectrometry}

LDI mass spectrometry was performed using a MALDITOF mass spectrometer (Autoflex III; Bruker-Daltonics, Leipzig, Germany) in reflectron mode. Before experiments, $\mathrm{WSi}_{2}$ chips were sonicated in isopropyl alcohol for $30 \mathrm{~s}$, washed in deionized water, and then dried under a gentle flow of nitrogen. A peptide solution was prepared in $10 \%$ methyl alcohol to a concentration of $10 \mathrm{pmol} / \mu \mathrm{L}$. It consisted of bradykinin 1-5 (monoisotopic mass: 572.7 Da), bradykinin 1-7 (756.9 Da), angiotensin II (1046.2 Da), substance P (1347.6 Da), and somatostatin (1637.9 Da). The chemicals were commercially obtained from Sigma-Aldrich and used without purification. For LDI experiments, a $0.2 \mu \mathrm{l}$ drop of the peptide solution ( 2 pmol) was taken and pipetted onto $\mathrm{WSi}_{2}$ surfaces, and then dried in ambient conditions. The $\mathrm{WSi}_{2}$ surfaces were moderately hydrophilic, so the sample drop somewhat spread on the surfaces. Thus, the actual sample consumption used to obtain a LDI mass spectrum was much lower than the applied quantity of 2 pmol.

\section{Results and Discussion}

Although efficient LDI from various nanostructured
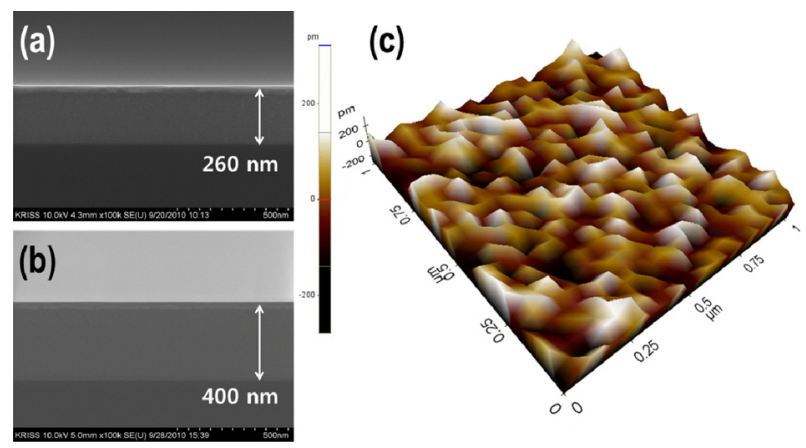

Figure 1. SEM (cross-section) and AFM images of $\mathrm{WSi}_{2}$ surfaces; (a) $260 \mathrm{~nm}$, (b) $400 \mathrm{~nm}$, and (c) $400 \mathrm{~nm}$ thick. surfaces is known, understanding of the LDI mechanism has been very limited. However, some of previous investigations recognized a possible contribution of laser-induced surface heating to the LDI process. ${ }^{3,10}$ It was viewed that the physical existence of surface nanostructures might provide heat conduction barriers for absorbed laser energy; thus, the absorbed energy is momentarily trapped in surface nanostructures, which gives rise to a rapid surface heating. Such a rapid increase in surface temperature is known to promote thermal desorption of thermally labile molecules, which thus can contribute to LDI of biomolecules. ${ }^{13}$

A recent work on a-Si demonstrated that a rapid surface heating for LDI could be achieved even without employing surface nanostructures by selecting appropriate surface materials. ${ }^{11}$ Because of the low thermal conductivity $(\mathrm{K}=$ $\left.5.5 \mathrm{~W} \mathrm{~m}^{-1} \mathrm{~K}^{-1}\right)$, the thermal diffusivity $\left(\kappa=\mathrm{K} / \mathrm{C}_{\mathrm{p}}\right)$ of a-Si is very small $\left(0.03 \mathrm{~cm}^{2} \mathrm{~s}^{-1}\right.$; heat capacity $\left(\mathrm{C}_{\mathrm{p}}\right)$ for a-Si $=1.65 \mathrm{~J}$ $\left.\mathrm{cm}^{-3} \mathrm{~K}^{-1}\right)$. During the period of laser irradiation $(\mathrm{t} \sim 7 \mathrm{~ns})$, the absorbed energy can diffuse only for about $90 \mathrm{~nm}$ (D) into the bulk substrate (depth of conductive heating: (D) $=(\kappa \mathrm{k} /$ $\pi)^{1 / 2}$ ). As the heat energy is localized near the surface due to the low thermal diffusivity, the surface temperature on a-Si can drastically increase to higher than $1000 \mathrm{~K}$ within $10 \mathrm{nsec}$. On the other hand, because of the higher thermal conductivity $\left(150 \mathrm{~W} \mathrm{~m}^{-1} \mathrm{~K}^{-1}\right)$ of c-Si, the transient increase in surface temperature is predicted to be only about $300 \mathrm{~K}$,

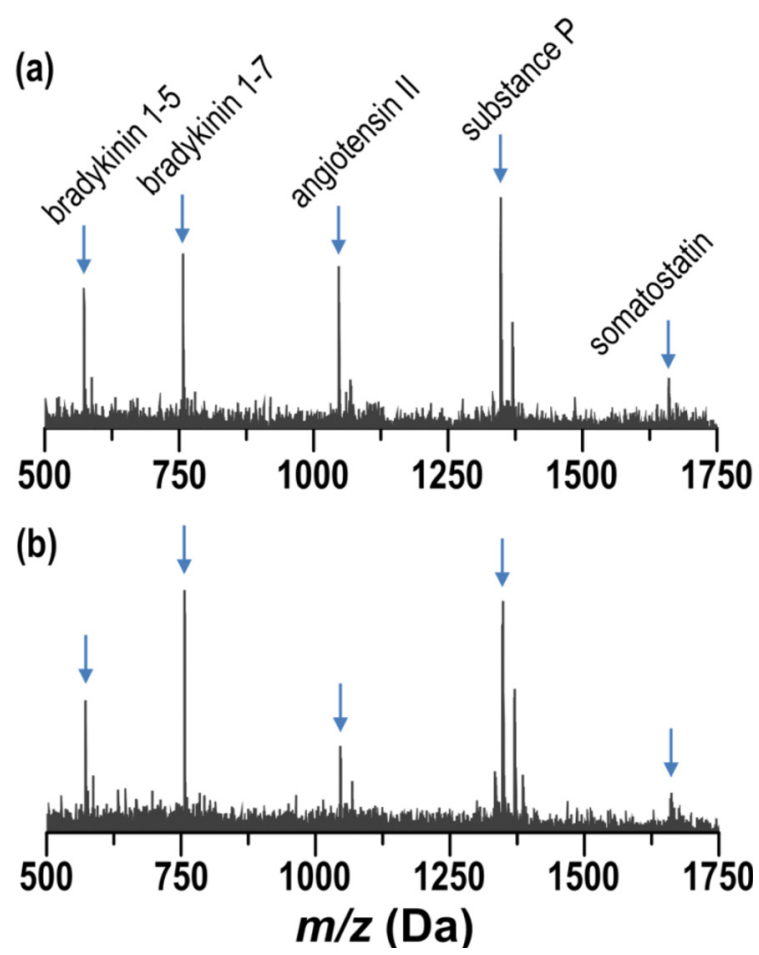

Figure 2. LDI mass spectra of a peptide mixture (bradykinin 1-5, bradykinin 1-7, angiotensin II, substance P, somatostatin (2 pmol each) from $\mathrm{WSi}_{2}$ surfaces (65 nm thick) (a) as prepared and (b) after high-temperature heat treatment. 


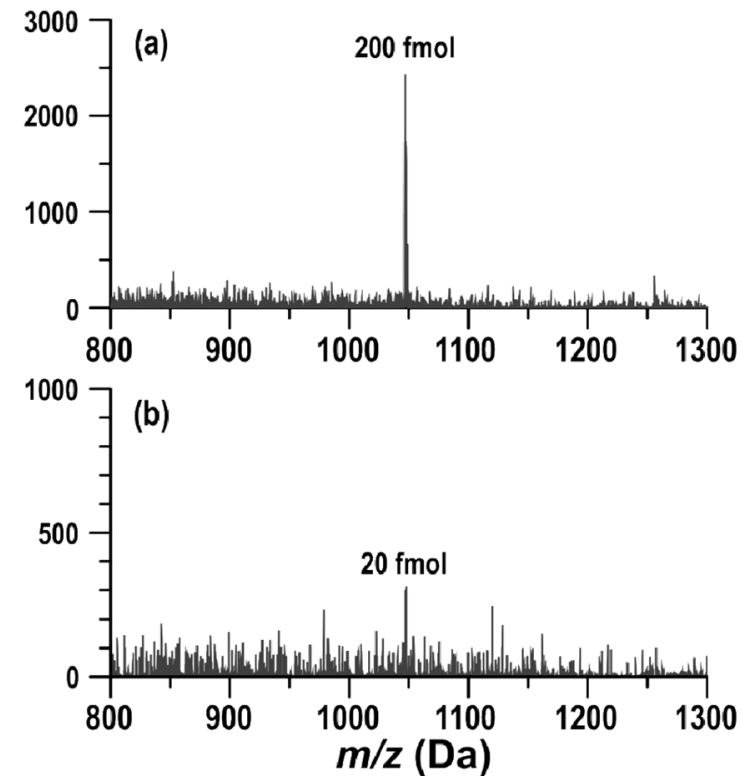

Figure 3. LDI mass spectra of angiotension II; (a) $200 \mathrm{fmol}$ and (b) $20 \mathrm{fmol}$.

much lower than on a-Si, which may explain that LDI of biomolecules on flat c-Si surfaces has not been successful before.

In this study, we examined $\mathrm{WSi}_{2}$ surfaces for flat LDI substrates. $\mathrm{WSi}_{2}$ possesses a moderate thermal conductivity of $18.3 \mathrm{~W} \mathrm{~m}^{-1} \mathrm{~K}^{-1}$. However, as typical in metal silicides, it has a very large heat capacity $\left(309.3 \mathrm{~J} \mathrm{~cm}^{-3} \mathrm{~K}^{-1}\right)$, which gives a much low thermal diffusivity of $0.0006 \mathrm{~cm}^{2} \mathrm{~s}^{-1}$ that predicts a conduction depth (D) of $11 \mathrm{~nm}$. Thereby, it can be suggested that the heat capacity of $\mathrm{WSi}_{2}$ surfaces might give us an opportunity for LDI of thermally labile molecules. In order to corroborate the idea, we examined LDI of peptides from $\mathrm{WSi}_{2}$ surfaces. Figure 1 displays SEM and AFM images for $\mathrm{WSi}_{2}$ surfaces used in this study. As shown in the AFM image, the surfaces do not possess any noticeable surface nanostructures, which would otherwise contribute to promoting the LDI process, possibly. We examined the LDI capability of $\mathrm{WSi}_{2}$ surfaces prepared in different conditions using a mixture of 5 peptides. For example, we examined $65 \mathrm{~nm}, 260 \mathrm{~nm}$, and $400 \mathrm{~nm}$ thick $\mathrm{WSi}_{2}$ surfaces before and after high-temperature heat treatment. However, within the conditions we varied, the results on LDI performance were not quite different. Figure 2 shows the LDI mass spectra of peptides obtained from $65 \mathrm{~nm}$ thick $\mathrm{WSi}_{2}$ surfaces with and without heat treatment. The results shown in Figure 2 clearly exhibit pronounced production of peptide ions from the flat surfaces of $\mathrm{WSi}_{2}$. It supports our postulation that the thermal mechanism is indeed a main driving force for LDI of thermally labile molecules from surfaces, where selecting surface materials with a large heat capacity can be a way to develop efficient
LDI plates. As shown in Figure 3, the LDI-on-WSi ${ }_{2}$ method also offers a good sensitivity of low femtomole levels ( $20 \mathrm{fmol}$ for angiotensin II), which is acceptable for peptide mass spectrometry as well.

\section{Conclusion}

We report that LDI on tungsten silicide $\left(\mathrm{WSi}_{2}\right)$ surfaces exhibits pronounced production of peptide ions. It covers a mass range up to $1600 \mathrm{Da}$. It is sensitive, which can ionize low femtomole levels of peptide molecules without assistance of matrices or surface nanostructures. The mechanism for LDI is viewed to be thermal, i.e. driven by laser-induced surface heating. It is most likely promoted by the thermal properties of $\mathrm{WSi}_{2}$ such as very large heat capacity, causing a rapid surface heating that favors intact desorption of thermally labile molecules like peptides. By demonstrating efficient LDI from flat surfaces of a material with a large heat capacity $\left(\mathrm{C}_{\mathrm{p}}\right)$, thus with a small heat diffusivity $(\kappa)$, we suggest that the thermal mechanism indeed plays a key role in LDI, which provides an important point of consideration in the future development of matrix-free LDI method.

\section{Acknowledgment}

The authors acknowledge support for this research from the Converging Research Center Program through the NRF, funded by MEST (2011K000887).

\section{References}

1. Peterson, D. S. Mass Spectrom. Rev. 2007, 26, 19.

2. Wei, J.; Buriak, J. M.; Siuzdak, G. Nature 1999, 399, 243.

3. Alimpiev, S.; Nikiforov, S.; Karavanskii, V.; Minton, T.; Sunner, J. J. Chem. Phys. 2001, 115, 1891.

4. Northen, T. R.; Yanes, O.; Northen, M. T.; Marrinucci, D.; Uritboonthai, W.; Apon, J.; Golledge, S. L.; Nordstrom, A.; Siuzdak, G. Nature 2007, 449, 1033.

5. Go, E. P.; Apon, J. V.; Luo, G.; Saghatelian, A.; Daniels, R. H.; Sahi, V.; Dubrow, R.; Cravatt, B. F.; Vertes, A.; Siuzdak, G. Anal. Chem. 2005, 77, 1641.

6. Walker, B. N.; Razunguzwa, T.; Powell, M.; Knochenmuss, R.; Vertes, A. Angew. Chem. Int. Ed. 2009, 48, 1669.

7. Walker, B. N.; Stolee, J. A.; Pickel, D. L.; Retterer, S. T.; Vertes, A. J. Phys. Chem. C. 2010, 114, 4835.

8. Nayak, R.; Knapp, D. R. Anal. Chem. 2010, 82, 7772.

9. Seino, T.; Sato, H.; Yamamoto, A.; Nemoto, A.; Torimura, M.; Tao, H. Anal. Chem. 2007, 79, 4827.

10. Shin, W. J.; Shin, J. H.; Song, J. Y.; Han, S. Y. J. Am. Soc. Mass Spectrom. 2010, 22, 989.

11. Kim, S. H.; Lee, A.; Song, J. Y.; Han, S. Y. J. Am. Soc. Mass Spectrom. 2012 (doi: 10.1007/s13361-012-0355-5)

12. Hsu, N.-Y.; Tseng, S. Y.; Wu, C.-Y.; Ren, C.-T.; Lee, Y.C.; Wong, C.-H.; Chen, C.-H. Anal. Chem. 2008, 80, 5203.

13. Daves, Jr., G. D. Acc. Chem. Res. 1979, 12, 359. 